\title{
Endovascular Approach for Arterial Occlusion in Blunt Trauma: Two Cases
}

\author{
Joongsuck Kim¹, Maru Kim², Hang Joo Cho², Yoo Dong Won ${ }^{3}$ \\ 'Department of Trauma Surgery, Cheju Halla General Hospital \\ ${ }^{2}$ Department of Trauma Surgery, Uijeongbu St. Mary's Hospital \\ ${ }^{3}$ Department of Radiology, Uijeongbu St. Mary Mary's Hospital
}

Arterial occlusion after blunt trauma is rare, but can be devastating to the supplied organs or limbs. Management can vary from simple observation to surgery, such as thrombectomy or bypass, which may not be structurally possible and can inflict additional damage. Thus, an endovascular approach is another management option. We present two cases of arterial occlusion after blunt trauma, one successfully treated by endovascular procedure and one failure of the endovascular treatment.

(Trauma Image Proced 2017(1):22-24)

Key Words: Endovascular procedures; Arteries; Injuries

\section{CASE 1}

A 22-year-old male arrived at the emergency department complaining of abdominal pain. He was struck on the mid abdomen by a slow moving truck. He was alert and his vital signs were stable. Contrastenhanced computed tomography (CT) of the abdomen demonstrated left renal artery occlusion along with total infarct of the left kidney (Fig. 1.). There was no gross hematuria. He was brought to the intervention room for renal angiography. Unfortunately, the artery was totally blocked, making passage of the guide wire impossible (Fig. 2.). Otherwise, there was no active bleeding, and he was observed. Follow-up CT performed 2 weeks later showed some collateral perfusion (Fig. 3.). Authors decided observation for the left kidney. The patient was discharged without complication.

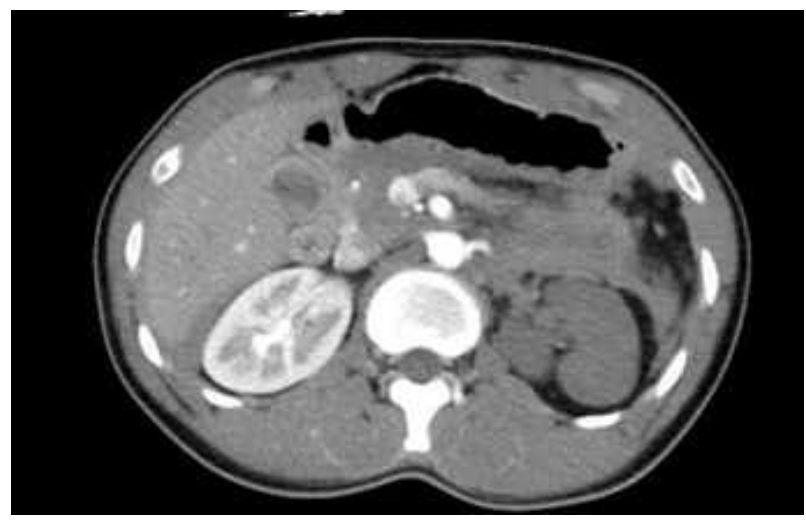

Fig. 1. Left renal artery occlusion

\section{CASE 2}

A 44-year-old male arrived at the emergency department complaining of pelvic and right leg pain

Received: April 2, 2017 Revised: April 28, 2017 Accepted: May 2, 2017

Correspondence to: Joongsuck Kim, Department Trauma Surgery, Cheju Halla General Hospital, 65, Doryeong-ro, Jeju-si, Jeju-do, Korea Tel: 82-64-740-5000, Fax: 82-64-743-3100, E-mail: jsknight68@daum.net

Copyright (c) 2017 Korean Association for Research, Procedures and Education on Trauma. All rights reserved.

(c)This is an open-access article distributed under the terms of the Creative Commons Attribution Non-Commercial License (http://creativecommons.org/ licenses/by-nc/4.0) which permits unrestricted noncommercial use, distribution, and reproduction in any medium, provided the original work is properly cited 


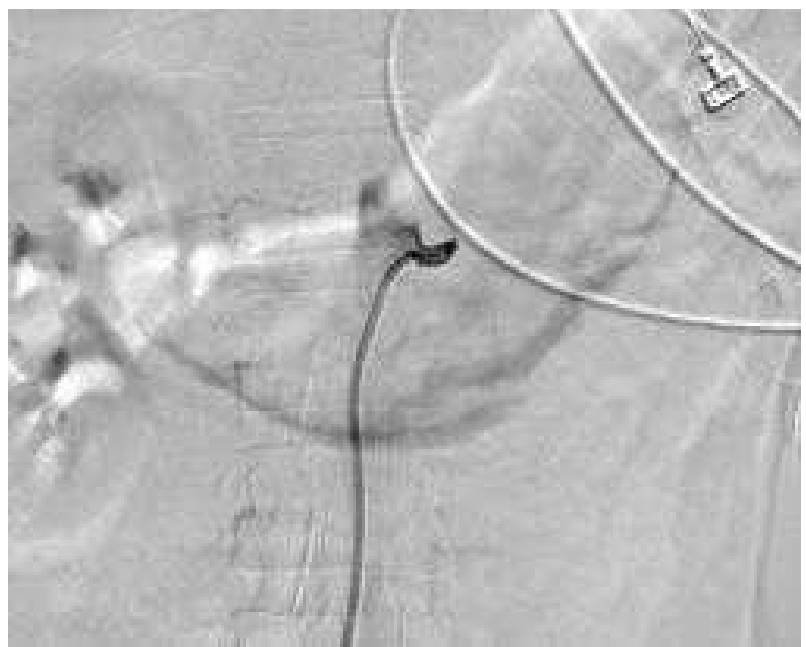

Fig. 2. Angiography shows total obstruction of the left renal artery.

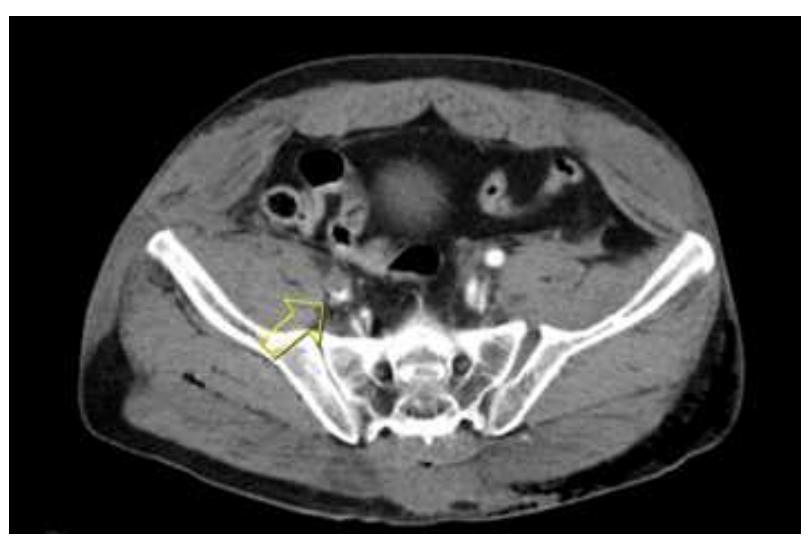

Fig. 4. CT shows left sacroiliac joint diastasis and right external iliac artery occlusion (arrow).

after falling from a $2 \mathrm{~m}$ height. He demonstrated unstable vital signs, which were stabilized after crystalloid infusion. Physical examination revealed an unstable pelvis and weak right femoral pulse. Contrastenhanced CT of the abdomen and pelvis demonstrated right inferior and superior rami fractures, left sacroiliac joint diastasis, and right external iliac arterial occlusion by thrombosis (Fig. 4.). Angiography revealed occlusion of the right external iliac artery, extending to the common femoral artery (Fig. 5.). The guide wire was able to pass and a stent graft was inserted. The post-stent angiography showed good arterial flow (Fig.
6.) and his femoral pulse returned to normal. His pelvis

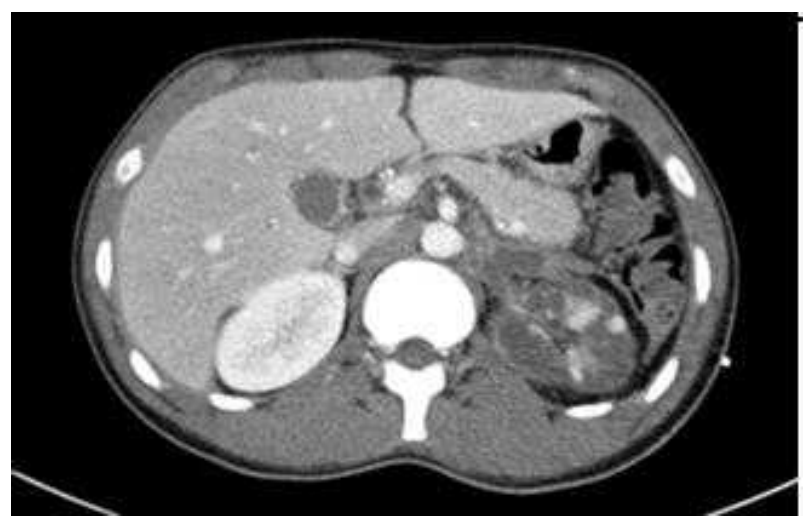

Fig. 3. Follow-up CT (2 weeks later) shows some collateral perfusion.

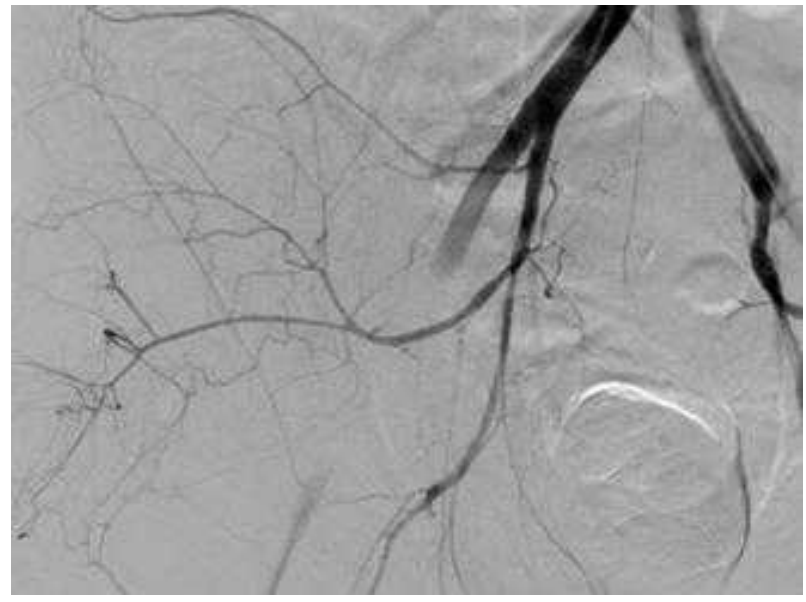

Fig. 5. Angiography shows right external iliac artery occlusion by thrombosis, extending to common femoral artery.

fracture was later managed by internal fixation, and 1 month later he was able to walk and was discharged without complication.

\section{DISCUSSION}

Arterial occlusions, especially those of peripheral arteries, after blunt trauma were reported 50 years ago (1). Ischemia secondary to trauma can be devastating. There are several reports of arterial occlusions with various managements, such as patch angioplasty (2) and treatment via an endovascular approach (3). Decisions 

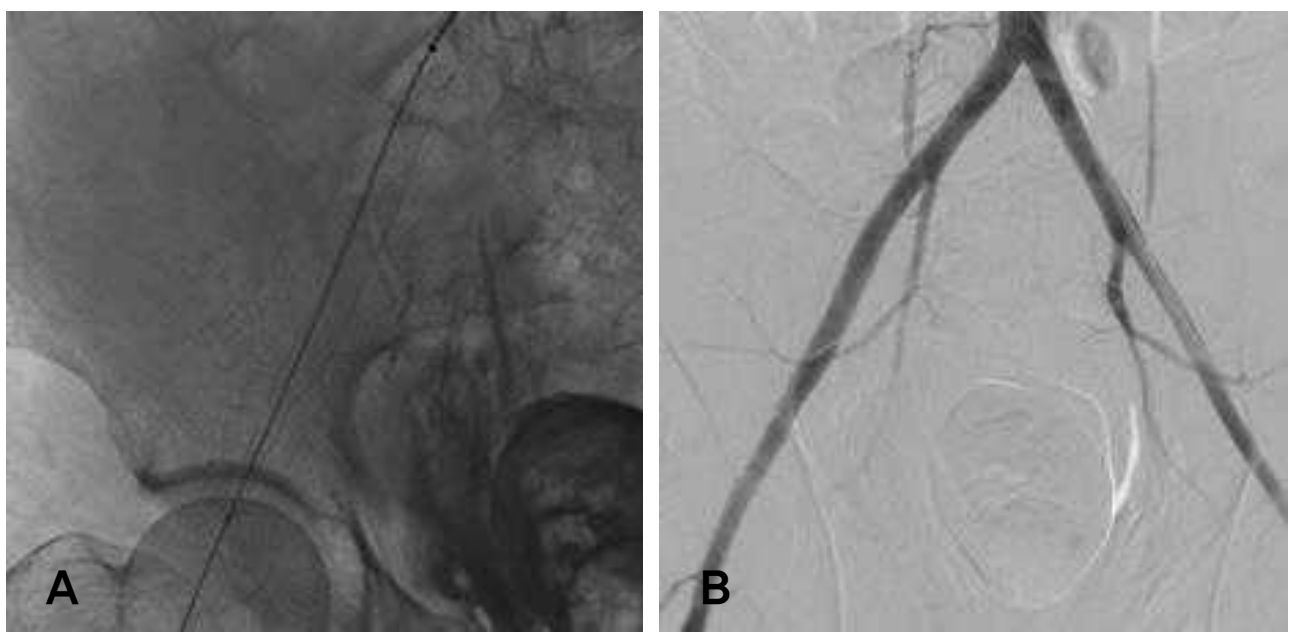

Fig. 6. (A) Guide wire was able to pass, and a 10-cm stent graft was inserted. (B) Post-stent angiography shows good arterial flow.

for treatment may differ depending on the inflicted artery, type and force applied, and timing. Renal artery occlusion, for instance, may not always require surgical revascularization when there is a functioning contralateral kidney (4). Trauma surgeons should be aware of various options, especially endovascular interventions when managing such patients.

\section{Conflict of Interest Statement}

No potential conflict of interest relevant to this article was reported.

\section{REFERENCES}

1. Ophir M, Shulemson M, Laufer M, Sinkower A. Acute peripheral arterial occlusion due to blunt traumatic rupture of the intima. Israel journal of medical sciences. 1967;4(4): 905-7.

2. Mogannam AC, Cubas RF, Gutierrez IM, Astudillo JA, Abou-Zamzam AM. Blunt Traumatic Occlusion of the Common Iliac Artery Repaired With Segmental Excision and Internal Iliac Artery Patch Angioplasty. Annals of vascular surgery. 2017;39:284. e1-. e4.

3. Mine T, Murata S, Yasui D, Tajima H, Kawamata H, Yokota $\mathrm{H}$, et al. Endovascular recanalization techniques for popliteal arterial occlusive injury with limb-threatening ischemia secondary to trauma. Acta radiologica short reports. 2014;3(1):2047981613518772.

4. Santucci RA, Wessells H, Bartsch G, Descotes J, Heyns C, McAninch $\mathrm{J}$, et al. Evaluation and management of renal injuries: consensus statement of the renal trauma subcommittee. BJU international. 2004;93(7):937-54. 